\title{
Alignment and Survey of the Elements in RHIC *
}

\author{
D. Trbojevic, P. Cameron, G. L. Ganetis, M. A. Goldman, R. Gupta, M. Harrison, M. F. Hemmer, \\ F. X. Karl, A. Jain, W. Louie, S. Mulhall, S. Peggs, S. Tepikian, R. Thomas, and P. Wanderer \\ Brookhaven National Laboratory \\ Upton, New York 11973, USA
}

\begin{abstract}
The Relativistic Heavy Ion Collider (RHIC) consists of two rings with cryogenic magnets at a $4.5 \mathrm{~K}$ operating temperature. Control of positions of the dipole and quadrupole cold masses (iron laminations) and the beam position monitors (BPM's) during production and installation is presented. The roll of the dipoles is controlled by a combination of rotating coil measurements with the surveying measurements. The center of the quadrupole magnetic field is obtained by direct measurement of the field shape within a colloidal cell placed inside the quadrupoles. Special attention is given to the triplet quadrupole alignment and determination of the field center position.
\end{abstract}

\section{Introduction}

Two rings of RHIC cross each other in the horizontal plane at six interaction regions. Dipoles and correctorquadrupole-sextupole (CQS) packages are installed inside the cryostats as they operate at a liquid helium temperature of $4.5 \mathrm{~K}$. During operation the magnet laminations are not accessible. In addition to collisions of heavy ions, the RHIC accelerators will do spin physics experiments with two proton beams. The spin physics puts additional constraints on the alignment of the RHIC magnets. The corrected closed orbit errors should be less than $1 \mathrm{~mm}$. The quadrupole and dipole rolls should be less than $1 \mathrm{mrad}$.

\section{The Global and Local Monument Networks in the RHIC Tunnel}

The sixfold symmetry of the RHIC accelerators imposed the primary network of twelve survey monuments on the tunnel floor[1]. A clear line of sight from the twelve platforms is available via a penetration through the tunnel berm. An additional tower is located at the geometrical center of RHIC. Clear lines of sight are available from this tower to each of the twelve peripheral instrument platforms. Two accelerators in the RHIC tunnel lie in a horizontal plane of an orthogonal Cartesian coordinate system where in the center of the rings the vertical axis points upward along the local gravity. The survey results of the primary network showed the error elipses for each of the twelve monuments to be between $0.32 \mathrm{~mm}$ and $0.70 \mathrm{~mm}$ on the major axis, and between $0.12 \mathrm{~mm}$ and $0.25 \mathrm{~mm}$ on the minor axis. The mean value on the major axis is 0.57 $\mathrm{mm}$ while the mean on the minor axis is $0.18 \mathrm{~mm}$. The

\footnotetext{
"Work performed under the auspices of the U.S. Department of Energy
}

secondary network in the tunnel was established with the floor monuments located at each half cell with a distance of 14.1 meters between them. Surveying in RHIC is performed by using a combination of PC compatible and Leica data collection hardware. Data collection is accomplished [2] using Zeos 386+ laptop computers with GeoNet software (ME5000.bas and DIRECT.bas), the Kern Mekometer for distance measuring and the Leica T3000K for direction observation. Vertical control is observed using the Leica NA 3000 bar code level and recorded on board removable REC module. Data are passed on the REC module through a GIF12 reader connected to a PC on an RS232C standard COM port.

\section{Control of the Dipole Center Positions During Production}

RHIC dipoles are produced by the Northrop-Grumman Corporation in Bethpage, New York. Properties of the BHIC dipoles as well as other details of the dipole production are presented in other papers at this conference [3][4][5]. The dipole aperture center is located by a combination of the optical survey of the four fiducials with the mechanical survey during production. The presurveying of every element in the RHIC accelerator is executed with a portable Leica ManCat system using a Compaq 486c portable computer and T3000. Data are presently passed via floppy disk. Four fiducials are welded to the ends of the laminations - the "cold mass fiducials". The optical survey also locates the cold mass fiducial positions with respect to the outside cryostat fiducials as well as the relation to the horizontal gravitational base plane of the dipole supporting stands. The field angle of the dipoles is determined by the rotating coil ("mole") measurements with respect to the same gravitational plane. These measurements of the magnetic field angle are performed at room temperature for all dipoles. The first thirty dipoles, as well as every tenth dipole after that, are also measured at a superconducting temperature of $4.5 \mathrm{~K}$ with currents of $660 \mathrm{~A}, 1450 \mathrm{~A}$, and $5000 \mathrm{~A}$. A correlation between the cold and warm measurements of the field angle shows a standard deviation of 0.20 mrad. The error in measurements as well as reproducibility, is also estimated to be $\pm 0.2 \mathrm{mrad}$. The dipole field angle obtained from the harmonic probe measurements is set to be perpendicular to the RHIC horizontal plane in the tunnel by a rotation around the axis which crosses the radius of the dipole curvature at $1 / 3$ of the average sagitta. The RHIC horizontal Cartesian plane perpendicular to the center gravitational vector is obtained after correction to the earth ellipsoidal curvature [1]. 


\section{A. Production Control of CQS Assemblies}

The three elements of the CQS assemblies are connected together inside the two welded "cold-mass" shells. The corrector, quadrupole, and sextupole vertical and radial positions are measured by the dial gauge indicator with electronic data transfer [5]. The quadrupole mechanical center is estimated from the measurements of the four upper and four lower notches in the laminations. The sextupole mechanical center is estimated from the positions of the two upper and two lower noches of the laminations close to the end of the sextupole. The cold masses are installed into the cryostat assemblies. Alignment of the CQS assemblies in the tunnel is based on the quadrupole center obtained by the ferrofluidic cell measurements.

\section{B. Direct Measurement of the Quadrupole and Sextupole Centers by Ferrofluidic Cell at $4.5 \mathrm{~K}$}

The development and other details of the ferrofluidic measurements were presented earlier [6]. The centers of the first thirty quadrupoles and sextupoles will be obtained by direct optical measurements of the quadrupole and sextupole magnetic field shapes seen inside the colloidal cell at a temperature of $4.5 \mathrm{~K}$. Figure 1 represents one of the results of the quadrupole center measurement with respect to the center line, related to the outside fiducials by the ManCat system.

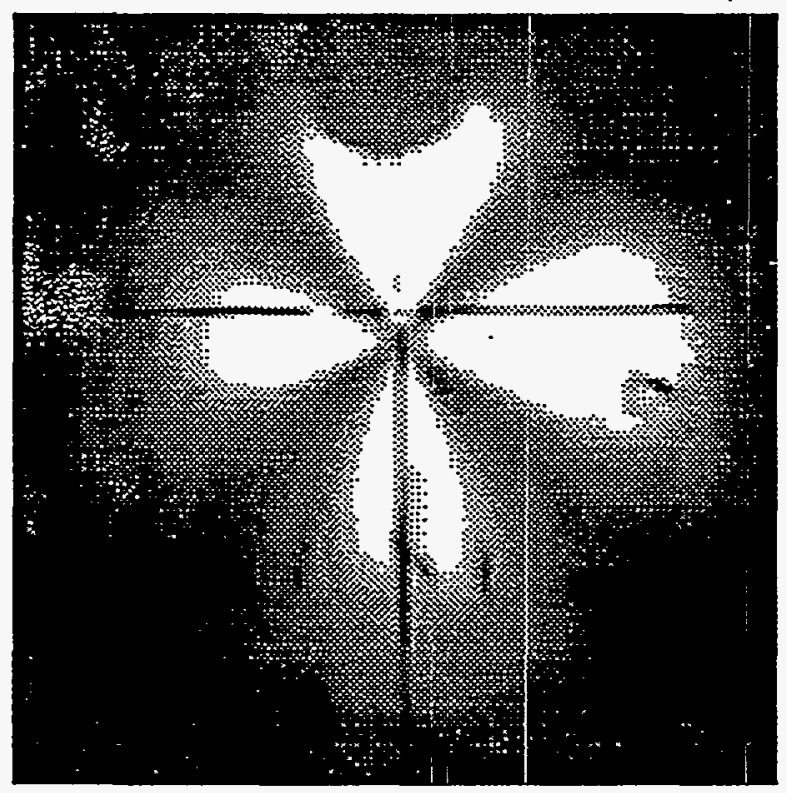

Figure. 1. Quadrupole field pattern viewed during colloidal cell measurements at $4.5 \mathrm{~K}$. Current through the quadrupole is $5000 \mathrm{~A}$.

Two polarizers are crossed to each other $\left(90^{\circ}\right)$ and placed at the opposite ends of the CQS assembly. The collimated light can not be seen with the telescope if there is no magnetic field around the cell with the colloidal solution. A cylindrical glass cell is filled with ferrofluidic colloidal solution [6]. When the quadrupole field is present the small particles in the colloidal solution orient themselves along the magnetic field lines producing the field picture at the telescope. Figure 2 is obtained from the sextupole measurements at $4.5 \mathrm{~K}$ with a current of $100 \mathrm{~A}$ through the sextupole coils (operating current of the sextupoles is $30 \mathrm{~A}$ ). The accuracy of the quadrupole magnetic field center obtained by the colloidal cell is $\pm 50 \mu \mathrm{m}$.

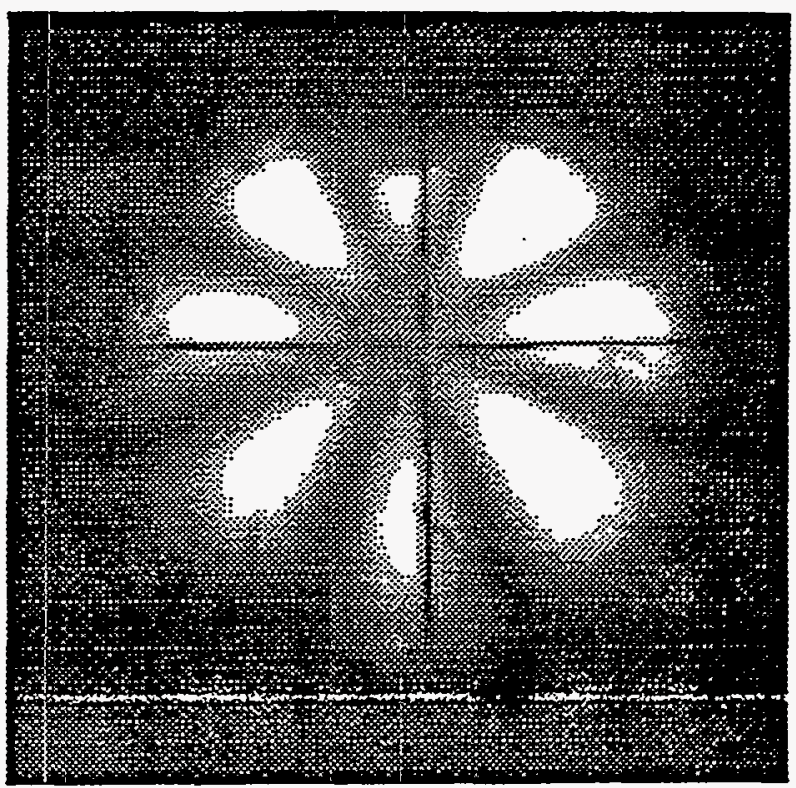

Figure. 2. Field patterns viewed in the colloidal cell inside the sextupole magnet at $4.5 \mathrm{~K}$. Current through the sextupole coils is $100 \mathrm{~A}$.

\section{Results from the Colloidal Cell Measurements of the Quadrupole Center at Room Temperature}

Most of the CQS assemblies will be measured at room temperature, by pulsing the superconducting coils with a current up to $300 \mathrm{~A}$ within 3 seconds. The colloidal cell quadrupole field shapes are obtained from the high resolution camera using the frame grabber. It takes 0.8 seconds for the ferrofluidic particles inside the colloidal solution to react to the outside magnetic field lines. Three pictures are taken during the 2 seconds flat top. Figure 3 shows the quadrupole field shape from the $300 \mathrm{~A}$ pulsing measurement. The calibration measurements at room temperature showed that one pixel is $50.8 \mu \mathrm{m}$ long. The accuracy of determining the center of the magnetic field could be fraction of a pixel.

\section{Triplet Quadrupole Alignment}

The collisions of the two beams at RHIC detectors "STAR" and "PHENIX" will have the maximum luminosity with the highest focusing of the triplet magnets. The twiss function $\beta^{*}=1 \mathrm{~m}$. At the storage conditions in RHIC the luminosity depends mostly on the magnetic field quality in the triplet quadrupoles as well as on the accuracy of their alignment. Special attention is given to the procedure and design of the triplet cryostat. Each quadrupole in the triplet cryostat will be aligned with an accuracy of 


\section{DISCLAIMER}

Portions of this document may be illegible in electronic image products. Images are produced from the best available original document. 


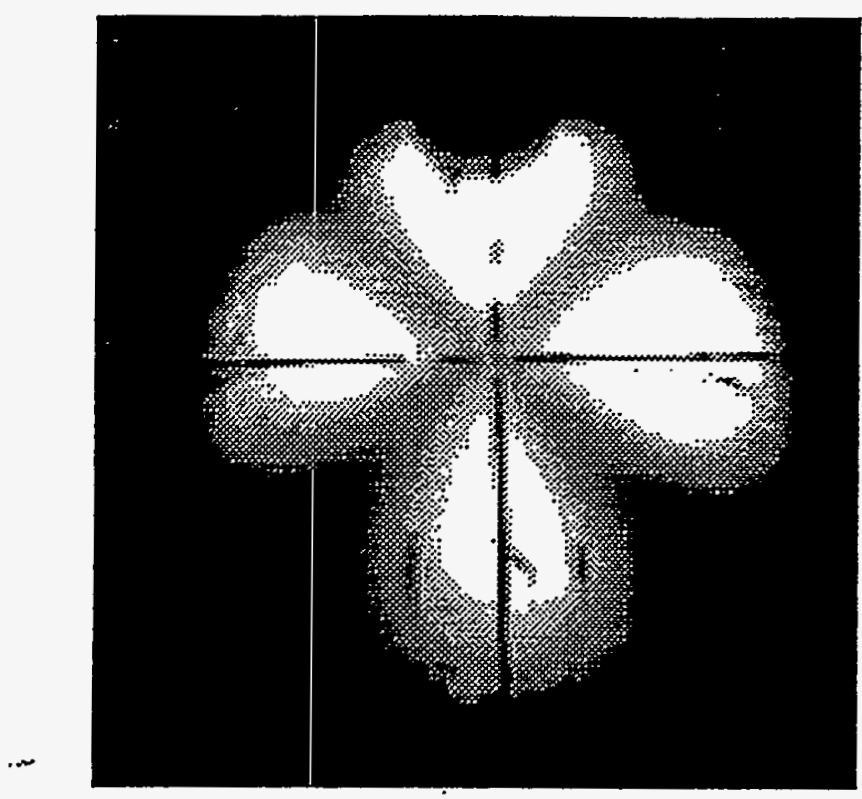

Figure. 3. Quadrupole field shape viewed in the colloidal cell during a measurement with a current through the quadrupole of $300 \mathrm{~A}$.

$\pm 50 \mu \mathrm{m}$ by using the colloidal cell technique. The triplet quadrupoles will be pulsed to produce a strong enough magnetic field to induce the field shape inside of the colloidal cell. The quadrupole field angle of the triplets is determined by the harmonic probe measurements with an estimated accuracy of \pm 0.2 mrad.

\section{The BPM Positions}

The beam position monitors (BPM's) are located inside the CQS assemblies at the sextupole end. The BPM position with respect to the outside fiducials is determined by direct measurement of the center with a pulsed antenna. A high frequency signal of $50 \mathrm{MHz}$ is sent to a rod placed close to the center of the BPM. The BPM plates provide signals which determine the position of the rod with respect to the plates. Part of the rod outside the CQS vacuum flange has two fiducials. These are used to determine the rod position with respect to the outside cryostat fiducials by using the already mentioned ManCat system. The accuracy of the BPM position with respect to the outside fiducials is estimated to be $\pm 0.13 \mathrm{~mm}$. During the calibration procedure it was found that the reproducibility and accuracy of the measurements was $\pm 50 \mu \mathrm{m}$.

\section{Conclusions}

The alignment errors of the RHIC arc quadrupole magnets in the RHIC accelerators are expected to be reduced to less than $\pm 0.25 \mathrm{~mm}$ due to the direct measurements of the quadrupole centers with respect to the outside cryostat fiducials by the optical colloidal cell measurements. The dipole and quadrupole magnetic field angles are obtained from harmonic probe measurements with an accuracy of $\pm 0.2 \mathrm{mrad}$. It is estimated that the rms roll errors of the dipoles will be less than $1 \mathrm{mrad}$ and in the case of the quadrupoles less than $0.5 \mathrm{mrad}$. The prefiducialization of every magnet in RHIC is automated with direct electronic data transfer and analyses. The best possible positions of the RHIC elements in the tunnel are obtained by a combination of the optical survey measurements, mechanical measurements, and harmonic probe field angle measurements.

\section{References}

[1] M.A. Goldman, "RHIC Reference Geometry", Brookhaven National Laboratory Internal Document, AD/RHIC/RD-43, August 1992.

[2] M. Hemmer, "RHIC Electronic Data Collection and Survey and Alignment Database", Brookhaven National Laboratory Internal Document, August 1993.

[3] P. Wanderer et al., "Construction and Testing of Arc Dipoles and Quadrupoles for the Relativistic Heavy Ion Collider (RHIC) at BNL", at this conference.

[4] J. Wei et al., "Field Quality Evaluation of the Superconducting Magnets of the Relativistic Heavy Ion Collider", at this conference.

[5] S. Mulhall et al., "Combined Element Magnet Production for Relativistic Heavy Ion Collider at BNL, at this conference.

[6] M.A. Goldman, R.E. Sikora, and T.J. Shea, "Preliminary Studies on a Magneto-Optical Procedure for Aligning RHIC magnets", IEEE Proceedings 1993, Washington DC May 17-20, pp.2916-2918.

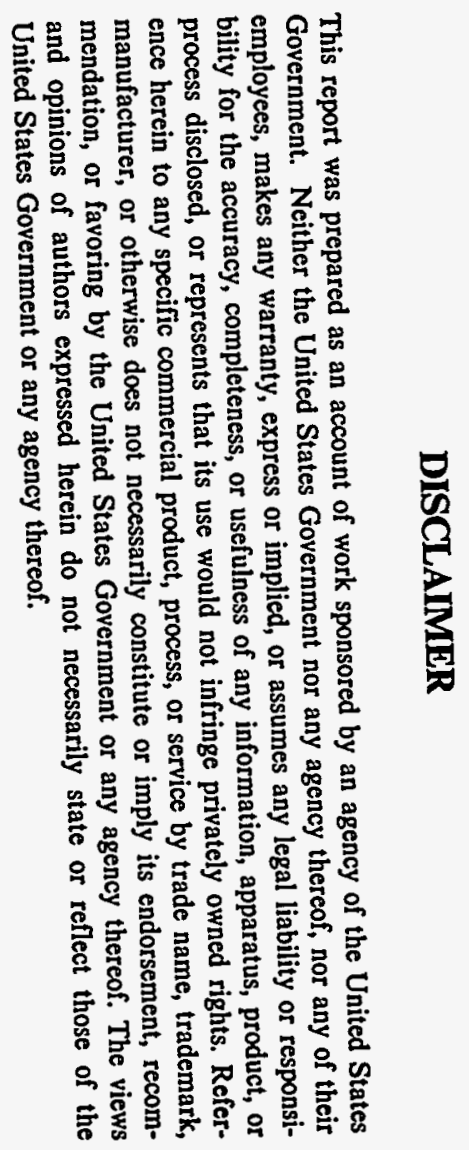

